\title{
An approach for leukemia classification based on cooperative game theory
}

\author{
Atefeh Torkaman $^{\mathrm{a}}$, Nasrollah Moghaddam Charkari ${ }^{\mathrm{b}, *}$ and Mahnaz Aghaeipour ${ }^{\mathrm{c}}$ \\ ${ }^{a}$ Department of Information Technology, Tarbiat Modarres University, Tehran, Iran \\ ${ }^{\mathrm{b}}$ Faculty of Electrical and Computer Engineering, Tarbiat Modarres University, Tehran, Iran \\ ${ }^{\mathrm{c}}$ Iranian Blood Transfusion Research Center, Tehran, Iran
}

\begin{abstract}
Hematological malignancies are the types of cancer that affect blood, bone marrow and lymph nodes. As these tissues are naturally connected through the immune system, a disease affecting one of them will often affect the others as well. The hematological malignancies include; Leukemia, Lymphoma, Multiple myeloma. Among them, leukemia is a serious malignancy that starts in blood tissues especially the bone marrow, where the blood is made. Researches show, leukemia is one of the common cancers in the world. So, the emphasis on diagnostic techniques and best treatments would be able to provide better prognosis and survival for patients. In this paper, an automatic diagnosis recommender system for classifying leukemia based on cooperative game is presented. Through out this research, we analyze the flow cytometry data toward the classification of leukemia into eight classes. We work on real data set from different types of leukemia that have been collected at Iran Blood Transfusion Organization (IBTO). Generally, the data set contains 400 samples taken from human leukemic bone marrow. This study deals with cooperative game used for classification according to different weights assigned to the markers. The proposed method is versatile as there are no constraints to what the input or output represent. This means that it can be used to classify a population according to their contributions. In other words, it applies equally to other groups of data. The experimental results show the accuracy rate of $93.12 \%$, for classification and compared to decision tree (C4.5) with $(90.16 \%)$ in accuracy.

The result demonstrates that cooperative game is very promising to be used directly for classification of leukemia as a part of Active Medical decision support system for interpretation of flow cytometry readout. This system could assist clinical hematologists to properly recognize different kinds of leukemia by preparing suggestions and this could improve the treatment of leukemic patients.
\end{abstract}

Keywords: Game theory, cooperative game, shapley value, classification, leukemia

\section{Background}

Hematological malignancies are a form of cancer that affect blood tissue, lymph nodes and bone marrow. As mentioned, the hematological malignancies include [14].

- Leukemia

- Acute lymphoblastic leukemia (ALL)

- Acute myelogenous leukemia (AML)

- Chronic myelogenous leukemia (CML)

- Chronic lymphocytic leukemia (CLL)

- Lymphoma:

${ }^{*}$ Corresponding author: N. Moghaddam Charkari, Tarbiat Modarres University, Department of Computer Science, Tehran, Iran. Tel.: +982182223301; E-mail: Charkari@modares.ac.ir.
- Hodgkin's disease (four subtypes)

○ Non-Hodgkin lymphoma (many subtypes)

- Multiple myeloma

Among them, Leukemia is a very common and serious cancer that starts in blood-forming tissues. Leukemia can be chronic (gets worse slowly) or acute (gets worse quickly). In acute leukemia, the blood cells are abnormal and cannot work properly. On the other hand, in chronic, the abnormal cells can continue their work in initial steps [Leukemia.emedty.com]. CLL usually affects people more than 55 years. CML is a form of cancer in which the bone marrow makes too many white blood cells and it affects on adult.

To analyze a suspected hematological malignancy, a complete blood count, blood film, bone marrow smear and the other diagnostic tools are essential. In this way, 
flow cytometry is one of the best techniques for the detection of hematological malignancy. Flow Cytometry is a method which generate immunophenotypic data. The ability to perform multiparametric analysis on individual cellular basis is a unique feature of Flow Cytometry. It offers some distinct advantages over competing immunophenotypic methods such as immunohistochemistry [28]. All hematopoietic cells conclude an expression of cluster of differentiation markers (CD markers). According to the markers values, different types of Leukemia could be classified. Generally, the interpretation of Flow Cytometry readouts is influenced by individual experience and knowledge. There is no doubt that diagnostic techniques and best treatments would be able to decrease the mortality rate from cancers. Recently, advances in diagnostic system have provided a new way of opportunities in cancer recognition. The introduction of Active Medical Decision Support System (A-MDSS) that aids the process of decision making in diagnosis problems has been one of the interesting subjects for many research groups. In our work, A-MDSS assists doctors and hematologists to properly recognize cancers and malignancies by preparing suggestions. In this regard, the probability of wrong diagnosis from many types of cancers could be decreased. In this case, the importance of A-MDSS is vital. A-MDSS is a subfield of Decision Support System (DSS), a set of interactive computer programs, which is designed to assist physicians and other health professionals with decision making tasks. For the first time, formal decision making theory was founded by VonNeuman and Morgerstern in 1940. Finally, medical decision making was proposed in 1980 and opened a new window to the realm of DSS. Fig. 1 shows a conceptual model of a Medical Decision Support System.

A novel distributed medical decision support system to help radiologists in the diagnosis of soft tissue tumors was introduced in [13]. This system is based on a distributed architecture with three specialized nodes: Radiologist Visual Interface, Information System and Decision Support Web-services. Sefion [20] employed a medical decision support system for asthmatic patient health care. The system helps physicians to control the chronic disease. A web based Medical Decision Support System applied for Neurological Diseases has been discussed in [24]. Development of medical decision support system for leukemia management was proposed in [9]. In this paper, in order to find the accurate knowledge model during predicting diagno- sis, four methods were applied; Rule-Based Reasoning (RBR), Case-Based Reasoning (CBR), Neural Network (NN), and Discriminant Analysis (DA). The average accuracy rate of classification based on the mentioned methods was not more than $70 \%$. Torkaman [26] suggested a recommender system for leukemia detection based on cooperative game theory. This system offers two candidates as diseases which are closer to the physician query by their minimum distances. Indeed, specialists are required to investigate the observations and determine the correct disease between the suggested candidates.

Machine learning (ML) algorithms have been efficiently employed to develop a decision support system. Machine learning is a suitable way to induction diagnostic and prognostic rules and solving special problems. The goal of machine learning is to program computers to use example data or past experience to solve a given problem. Many successful applications of machine learning exist in different applications, including systems that analyze past sales data to predict customer behavior, recognize faces or spoken speech, optimize robot behavior, extract knowledge from bioinformatics data, and data classification. [2]

Data classification is one of the most interesting subjects in decision science. In many types of DSS, such as A-MDSS, Data classification techniques have been considered.

A well-planned data classification system makes essential data easy to find. Today, many problems such as disease diagnosis, image recognition, and credit evaluation employ different types of classification techniques [17].

\subsection{Problem statement}

An expert usually requires interpreting the data gathered from Flow Cytometry and recognizing that a given sample belongs to which category of cancers. Right evaluation of data and making decisions properly are the most important items in cancer diagnosis. In this way, MDSS in medical diagnosis has been definitely applicable. Classification systems in MDSS not only help experts to make a better decision, but also minimize some possible errors. In this regard, a systematic approach is required to precisely classify the mentioned cancers according to their markers. In this paper a classification method based on cooperative game theory is investigated to assign diseases to the right classes. 


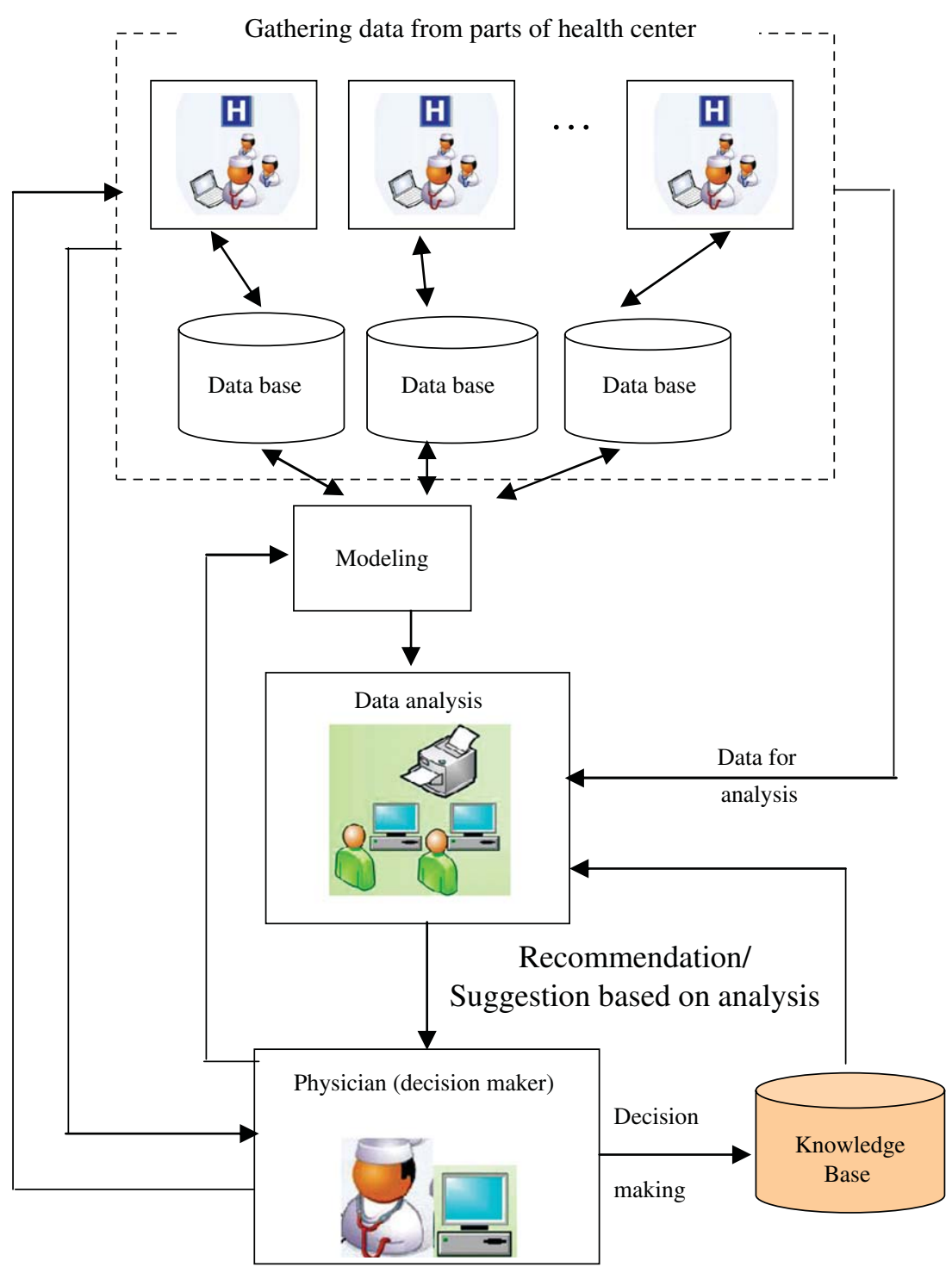

Fig. 1. A conceptual model of a Medical Decision Support System.

\section{Related work on cancer diagnosis}

There exist different studies on medical diagnosis of various types of cancers which most of them applied learning algorithms for data classification. Among them, artificial neural network (ANN) and linear classifier, like support vector machine, are most commonly used.

Feature selection were proposed by $[1,11,15,19]$ to reduce the size of feature vectors and to determine the good subsets of input variables. The task of feature selection is to choose a subset of input variable which maximizes the performance of the classifier. For example in [11] a better identification tool in fine needle aspiration cytology (FNAC) was developed to classify the breast cancer pattern. In this regard, multivariate adaptive regression splines (MARS) used to determine a good subset of input variables of a neural network model. Hence, the classification accuracy increased based on the proposed hybrid methodology. A new hybrid method based on fuzzy-artificial immune system and K-nn algorithm was proposed for breast cancer 
diagnosis in [19]. As we know, K-nn algorithms are known for their efficiency and simplicity in machine learning. But, for large data sets, these algorithms are very time consuming. In medical diagnosis, time and classification accuracy are two important issues. So, an artificial intelligence algorithm was proposed to reduce the size of input variables. An expert system for detecting breast cancer based on association rules (AR) and neural network was developed in [15]. In this study, $\mathrm{AR}$ is used for reducing the dimension of breast cancer dataset and NN for intelligent classification. In [1], Support vector machines (SVM) applied for breast cancer diagnosis. To select the optimal input of feature subset for SVM and to have a good prediction and less computational time as well, F-Score was employed. Chang and Chen tried to increase the quality of dermatologic diagnosis [10]. In this way, decision tree and neural network was applied. Bollschweiler showed that the preoperative diagnosis of lymph node metastases (LNM) as a difficult task. So, an artificial neural network was applied to predict LNM in patients with gastric cancer [5].

In [21] SVM has been applied to breast cancer classification. As the differentiation of a malignant and benign tumor is a complex and time consuming task, an automated technique that could help the physician was proposed. In this paper a group of images using Fine Needle Aspiration (FNA) biopsies of the breast was considered. In order to recognize the difference between malignant and benign class, SVM was applied. Various studies such as [3, 4, 16] have suggested the development of game theory as a fundamental paradigm to deal with the interactions between genes, cells and the outer environment. Bellomo and Delitala used stochastic game theory to modeling mutations, onset, progression and immune competition of cancer cells [4]. An Evolutionary Game was employed to describe the evolution of tumor cell populations with interactions among cells [3]. Mansury proposed Evolutionary game theory in an agent-based brain tumor model [16]. In [12], a game theoretical approach introduced to solve the classification problem in gene expression data analysis.

\section{Cooperative Game Theory and the Shapley value}

In this paper, we propose cooperative game and the Shapley value [22] to classify diseases. As we know, Game theory is divided into two classes, noncooperative and cooperative games. These classes differ in how they formalize interdependence among the players [6] (See Fig. 2).

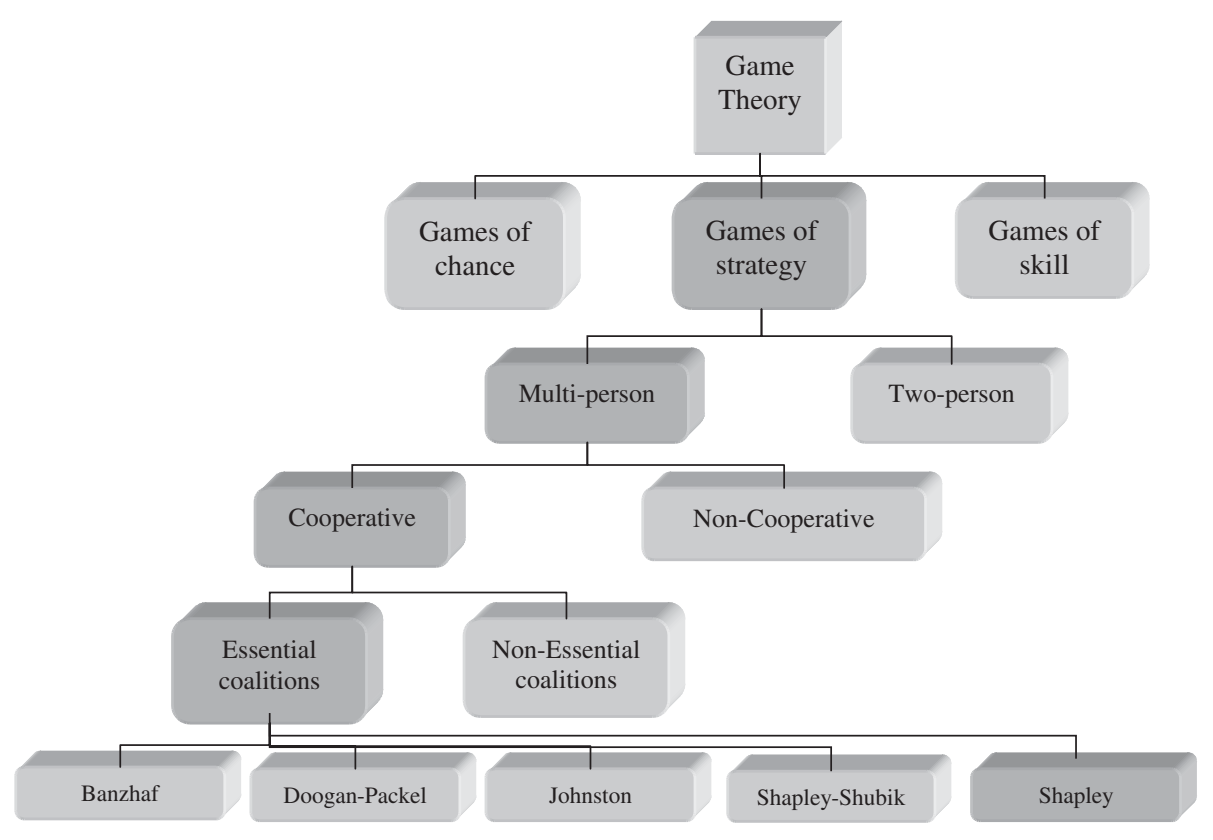

Fig. 2. A general game classification. 
In general, a cooperative game consists of a set of players, and a characteristic function which specifies the value created by different subsets of the players in the game. Let $\mathrm{N}=\{1,2, \ldots, \mathrm{n}\}$ be the (finite) set of players and $\mathrm{v}(\mathrm{S})$ as the characteristic function which is the value created when the members of $S$ come together and interact. The values concede to the contribution of the players in achieving a high payoff. A cooperative game with transferable utility (TU-game) is an ordered pair $(\mathrm{N}, \mathrm{v})$ consisting of the player set $\mathrm{N}$ and the characteristic function $v: 2^{N} \rightarrow \Re$ with $v(\phi)=0$ [7]. $\mathrm{v}(\mathrm{S})$ is interpreted as the maximal worth, or cost savings, where $\mathrm{S}$ can be obtained during cooperation.

A coalition is a group of players $T \subseteq N$ where it's value is found by $\mathrm{v}(\mathrm{T})$. A TU-game $(\mathrm{N}, \mathrm{v})$ is a $[0,1]$ game when $v: 2^{N} \rightarrow[0,1]$.

A TU-game is simple if $v(S) \in\{0,1\}$ and $\mathrm{v}(\mathrm{S})=1$ $V(S)=1 \Leftrightarrow S$ considered as winning where $v(S)=$ $0 \Leftrightarrow S$ is losing.

The payoff vector or allocation $(X i)_{i \in N} \in \mathfrak{R}^{N}$, assigns the amount $X_{i}$ to player $i \in N$ in a TU-game $(\mathrm{N}, \mathrm{v})$. This allocation is efficient if $\sum_{i \in N} X_{i}=v(N)$.

A solution for a class of TU-games is a function $\psi$ that assigns to every TU-game in the class, a payoff vector $\psi(v)$. The Shapley value, introduced by Shapley [22] is a well-known solution for TU-games. It assigns its average marginal contribution to each player over all the possible orderings, i.e. permutations of the players [12]. Given that $\mathrm{G}=(\mathrm{N}, \mathrm{v})$ is a game, the Shapley value assigns to player $i \in N$ will be denoted as; $\phi_{i}(v)=\frac{1}{n !} \sum_{\pi}(v(P(\pi ; i) \cup\{i\})-v(P(\pi ;\{i\})))(1)$

Where $\pi$ is a permutation of the players and $P(\pi ; i)$ is the set of players that precede player $i$ in the permutation $\pi$.

If two players $\mathrm{i}, \mathrm{j}$ are symmetric i.e. $v(S \cup\{i\})=$ $v(S \cup\{j\}), \forall S \subseteq N \backslash\{i, j\}$ then $\phi_{i}(v)=\phi_{j}(v)$.

If a player i has null marginal contributions i.e. $v(S \cup$ $\{i\})=v(S), \forall S \subseteq N \backslash\{i\}$, then $\phi_{i}(v)=0$.

As we know, classification is a procedure in which data items are placed into groups (classes) based on quantitative information on one or more characteristics inherent in the items. A classifier $h: X \rightarrow Y$, maps an object $x \in X$ to its classification label $y \in Y$.

\section{Method}

Now, the goal of this paper is to introduce a method which classifies different types of leukemia according to the value of their CD markers. In other words, each CD markers (x) should be mapped to its classification label (y) (weights, which are found by the use of cooperative game). A block diagram of the implemented system, as a part of A-MDSS, is shown in Fig. 3. There are two major steps for classification different kinds of leukemia; Training phase and diagnosis phase (Testing phase).

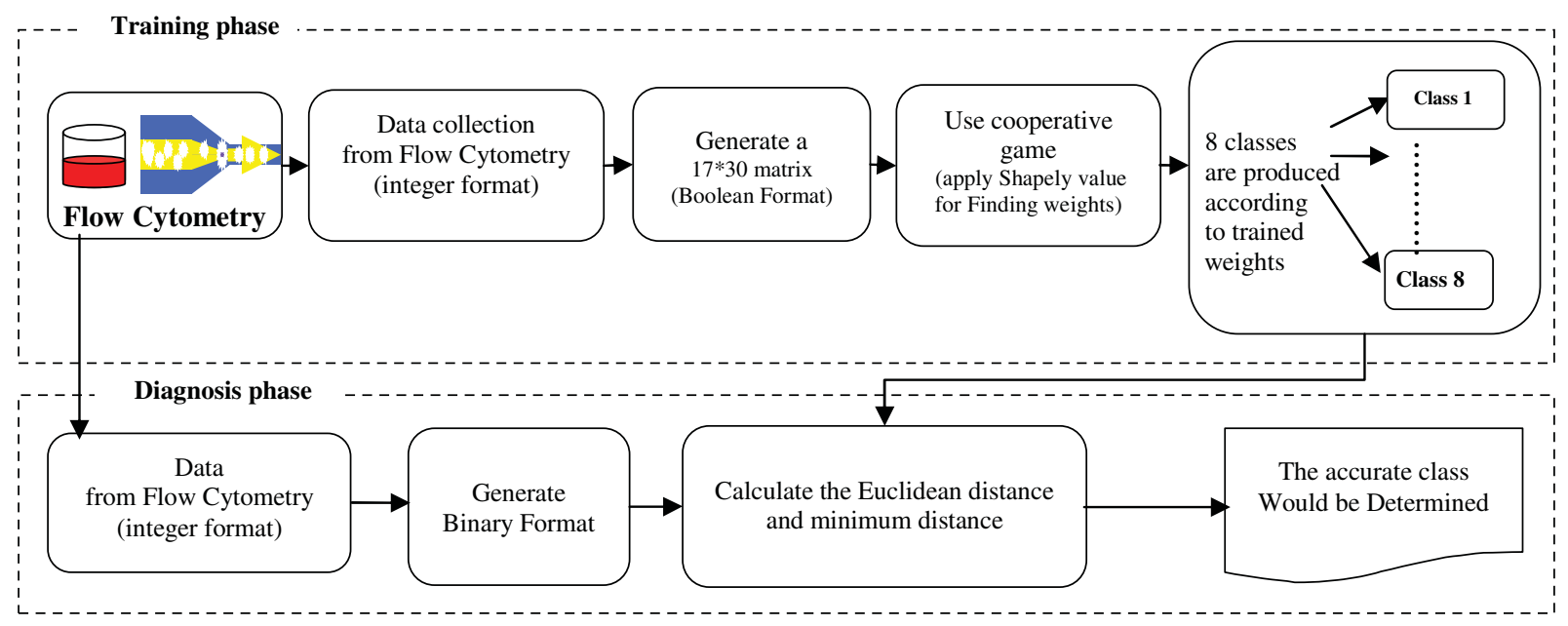

Fig. 3. System proposed based on cooperative game to classify samples. 


\subsection{Training phase}

As previously mentioned, precise classification method causes an accurate suggestion to the oncologist in diagnosis step. In order to classify properly, cooperative game theory and the use of Shapley value are applied.

In training phase, the collected data from Flow Cytometry provide the initial samples.

In our activity these data are considered as two main classes; ALL and AML which have 4 subclasses respectively ${ }^{1}$.

ALL can be either T or B lineage. In this study, we work on B lineage class. B-lineage ALL cells express the main antigens called CD19, CD20, CD34, CD45, HLA-DR, and/or CD10. Pro-B ALL is the propagation of the blasts of the $\mathrm{B}$ lineage.

B-lineage ALL is further classified into subtypes as follows;

Pro B ALL: Pro B Acute Lymphoid Leukemia

Early pre B ALL: Early Pre B Acute Lymphoid Leukemia

Pre B ALL: Pre B Acute Lymphoid Leukemia

B Cell ALL: B Cell Acute Lymphoid Leukemia

Acute myeloid leukemia (AML), also known as acute myelogenous leukemia, is a neoplasm of the myeloid linage of blood cells. This cancer characterized by the rapid growth of abnormal white blood cells that aggregate in the bone marrow. AML express the main antigens called CD13, CD14, CD33, CD34, CD41, CD45, CD64, CD71, CD117, GlycophorinA, and HLA DR.

AML is classified into subtypes as follows;

- AML M3: Acute Promyelocitic Leukemia M3 (APL)

- AML Non M3: Acute Myeloid Leukemia Non M3 (M1 M2)

- AML M4/M5: Acute Myelomonoblastid Leukemia M4 M5

- AML M6: Erythroid Leukemia M6

- AML M7: Acute Megakaryocylic Leukemia M7

(In IBTO there were few samples of AML M3 (APL), which were not investigated in this activity.)

\footnotetext{
${ }^{1}$ Complete panels of antigen expression are available on a few web sites: Hematopathologic Phenotypes Made Mockingly Simple, University of Washington hematopathology lab web site., from the Rubnitz/Pui article in The Oncologist.
}

Initially, these data are in integer form. First, data are converted to Boolean format, where 1 referring to those CD markers which are more than 20 in value and 0 otherwise. However, this is a critical task as the value of each CD markers, may deeply influence on the data classes. In practice, the threshold value is chosen by the hematologist experts.

Collected data are stored in matrices which consist of 17 rows defined as $\mathrm{N}$, referring to the $\mathrm{CD}$ markers (attributes) and 30 columns defined as $\mathrm{M}$; represent different samples of diseases in the training phase.

For example the conversion matrix of Pro B ALL is shown in Fig. 4.

In order to select the set of CD markers (attributes) in classifying samples, we use cooperative game, with each marker acting as a player. So that the number of players become $\mathrm{N}^{*} \mathrm{M}$. In this way, we have used Shapley value (Eq. (1)) to determine the weights and values of each marker (player). To the best of our knowledge, one of the main advantages of the Shapley value is its ability to provide a fair and unique solution. The fairness property determines the real portion of each player in the game. The usage of the Shapley value for our goal can be justified by its axiomatic qualities as mentioned bellow;

Axiom 1. Pareto optimality or Normalization: For any game $(\mathrm{N}, v)$, it describes that $\sum_{i \in N} \phi_{i}(v)=v(N)$.

Where $\mathrm{N}$ is the number of players, $\mathrm{v}$ is the value function for the coalitional game and $\phi_{i}(v)$ is the expected payoff to player i.

This axiom implies that the portion of a data set which is accurately divided between different markers.

Axiom 2. Permutation invariance or symmetry: For any $(\mathrm{N}, v)$ with ermutation $\pi$ on $\mathrm{N}, \phi_{i}(v)=\phi_{\pi(i)}(\pi v)$.

It determines that any arbitrary renaming or reordering of the markers will not change the final value.

Axiom 3. dummy-property: For any $(\mathrm{N}, v)$ where $v(S \cup$ $\{i\})=v(S), \forall S \subseteq N \backslash\{i\}$ then $\phi_{i}(v)=0$.

It indicates that a marker that does not influence a disease certainly receives zero value.

Accordingly, we determine the precise value of each marker and its importance in causing the disease. Then, we use them as primary knowledge in our expert system to classify data. By this way, 8 classes of the disease will be found. The number of classes corresponds to the number of diseases. We emphasize the role played by a set of markers in correlation with groups. 


\begin{tabular}{|l|l|l|l|l|l|l|l|l|l|l|l|l|l|l|l|l|l|l|l|l|l|}
\hline \multicolumn{1}{|c|}{ Samples } & S1 & S2 & S3 & S4 & S3 & S6 & S7 & S8 & S9 & S10 & S11 & S12 & S13 & S14 & S15 & S16 & S17 & S18 & S19 & S20 & $\cdots$ \\
CD Markers & & & & & & & & & & & & & & & & & & & & \\
\hline HLA-DL & 1 & 1 & 1 & 1 & 1 & 1 & 1 & 1 & 1 & 1 & 1 & 1 & 1 & 1 & 1 & 1 & 1 & 1 & 1 & 1 & $\cdots$ \\
\hline CD3 & 0 & 0 & 0 & 0 & 0 & 0 & 0 & 0 & 0 & 0 & 0 & 0 & 0 & 0 & 0 & 0 & 0 & 0 & 0 & 0 & $\cdots$ \\
\hline CD20 & 0 & 0 & 0 & 0 & 0 & 0 & 0 & 0 & 0 & 0 & 0 & 0 & 0 & 0 & 0 & 0 & 0 & 0 & 0 & 0 & $\cdots$ \\
\hline CD5 & 0 & 0 & 0 & 0 & 0 & 0 & 0 & 0 & 0 & 0 & 0 & 0 & 0 & 0 & 0 & 0 & 0 & 0 & 0 & 0 & $\cdots$ \\
\hline CD10 & 0 & 0 & 0 & 0 & 0 & 0 & 0 & 0 & 0 & 0 & 0 & 0 & 0 & 0 & 0 & 0 & 0 & 0 & 0 & 0 & $\cdots$ \\
\hline CD19 & 1 & 1 & 1 & 1 & 1 & 1 & 1 & 1 & 1 & 1 & 1 & 1 & 1 & 1 & 1 & 1 & 1 & 1 & 1 & 1 & $\cdots$ \\
\hline CD13 & 0 & 0 & 0 & 0 & 0 & 0 & 0 & 0 & 0 & 0 & 0 & 0 & 0 & 0 & 0 & 0 & 0 & 0 & 0 & 0 & $\cdots$ \\
\hline CD33 & 0 & 0 & 0 & 0 & 0 & 0 & 0 & 0 & 0 & 0 & 0 & 0 & 0 & 0 & 0 & 0 & 0 & 0 & 0 & 0 & $\cdots$ \\
\hline CD34 & 1 & 1 & 0 & 0 & 1 & 1 & 1 & 1 & 0 & 1 & 1 & 1 & 0 & 0 & 1 & 1 & 1 & 1 & 0 & 1 & $\cdots$ \\
\hline CD14 & 0 & 0 & 0 & 0 & 0 & 0 & 0 & 0 & 0 & 0 & 0 & 0 & 0 & 0 & 0 & 0 & 0 & 0 & 0 & 0 & $\cdots$ \\
\hline CD45 & 1 & 1 & 1 & 1 & 1 & 1 & 1 & 0 & 1 & 1 & 1 & 1 & 1 & 1 & 1 & 1 & 1 & 0 & 1 & 1 & $\cdots$ \\
\hline CD117 & 0 & 0 & 0 & 0 & 0 & 0 & 0 & 0 & 0 & 0 & 0 & 0 & 0 & 0 & 0 & 0 & 0 & 0 & 0 & 0 & $\cdots$ \\
\hline CD64 & 0 & 0 & 0 & 0 & 0 & 0 & 0 & 0 & 0 & 0 & 0 & 0 & 0 & 0 & 0 & 0 & 0 & 0 & 0 & 0 & $\cdots$ \\
\hline CD31 & 0 & 0 & 0 & 0 & 0 & 0 & 0 & 0 & 0 & 0 & 0 & 0 & 0 & 0 & 0 & 0 & 0 & 0 & 0 & 0 & $\cdots$ \\
\hline OlycophorinA & 0 & 0 & 0 & 0 & 0 & 0 & 0 & 0 & 0 & 0 & 0 & 0 & 0 & 0 & 0 & 0 & 0 & 0 & 0 & 0 & $\cdots$ \\
\hline CD41 & 0 & 0 & 0 & 0 & 0 & 0 & 0 & 0 & 0 & 0 & 0 & 0 & 0 & 0 & 0 & 0 & 0 & 0 & 0 & 0 & $\cdots$ \\
\hline CD61 & 0 & 0 & 0 & 0 & 0 & 0 & 0 & 0 & 0 & 0 & 0 & 0 & 0 & 0 & 0 & 0 & 0 & 0 & 0 & 0 & $\cdots$ \\
\hline
\end{tabular}

Fig. 4. Conversion $\mathrm{N} \times$ Mmatrix of Pro B ALL.

Let $B \in\{0,1\}^{n \times k}$ be a boolean matrix, where $n$ denotes the number of CD markers and $\mathrm{k}$ is the number of samples (from patients' results).

It is simply possible to calculate Shapley value by Eq. (1) for matrix B; determines the weight of each CD marker. Shapley value assigns a value to each marker according to its participation in the game. When the weights are found, CD markers will be trained by the mentioned weights. Table 1 is an example where Shapley values assign different weights to each marker of B-Cell-All and Aml-M4-M5.

One of the interesting ways to show the weights is to use a graphical tool called Hinton diagram [25]. The Hinton diagram provides a qualitative display of the values in a data matrix (normally a weight matrix). Each value is represented by a square whose size is associated with the magnitude of positivity within a sample and indicates the existence of a marker in a malignancy. The corresponding Hinton Diagram is shown below in Fig. 5. In this diagram, the weights means the Shapley values were assigned to each marker in a malignancy. Horizontal axis refers to CD mark- ers and the vertical axis is the different kinds of Leukemia.

At a glance, the Hinton diagram tell us which markers are extremely exist or not in a malignancy. For example, in Table 1, the HLA/DR, CD20, CD10, CD19, CD45 have approximately the same values in the B Cell malignancy. So, in Hinton diagram, each one has a square as size as the others. In contrast, CD3 by a little value has a small size square. This means that all the previous markers exist in that malignancy and CD3 has the minimum size in this group.

CD19 expresses in AML non M3 represents as an aberrant marker because CD19 is a B cell marker. CD19 is one of the markers, which has value in diagnosis of biphenotypic acute leukemia.

\subsection{Diagnosis phase (Testing)}

In this phase, new Flow Cytometry samples are prepared (Fig. 3). The Collected data provide the initial samples for test phase. These data are separated from the Training phase. Similarly, Integer data are con- 
Table 1

Shapley value assigns different values (weights) to each marker of B Cell All and Aml M4 M5

\begin{tabular}{lcc}
\hline CD-Markers Classes & B-Cell-All & Aml-M4-M5 \\
\hline HLA-DL & 0.3647 & 0.2933 \\
CD3 & 0.0294 & 0 \\
CD20 & 0.3647 & 0 \\
CD5 & 0 & 0 \\
CD10 & 0.2765 & 0 \\
CD19 & 0.3647 & 0 \\
CD13 & 0 & 0.2639 \\
CD33 & 0 & 0.2933 \\
CD34 & 0 & 0.1345 \\
CD14 & 0 & 0.2227 \\
CD45 & 0.3647 & 0.2933 \\
CD117 & 0 & 0 \\
CD64 & 0 & 0.2639 \\
CD71 & 0 & 0 \\
GlycophoinA & 0 & 0 \\
CD41 & 0 & 0 \\
CD61 & 0 & 0 \\
\hline
\end{tabular}

verted to Boolean format; as mentioned in Training phase.

Then, in order to measure the similarity between the new sample and the trained classes, we have used simple Euclidean distance which is an efficient similarity measurement.

$$
\begin{aligned}
d_{i j} \equiv & \left(\sum_{k=1}^{p}\left(x_{i k}-x_{j k}\right)^{2}\right)^{1 / 2} \\
& \left\{\begin{array}{l}
x_{i k} ; \text { New sample } \\
x_{j k} ; \text { The Trained class }
\end{array}\right.
\end{aligned}
$$

The minimum distance between the new sample and the trained classes is calculated. Accordingly, one can determine the accurate class for any diseases.

In this phase, 20 new samples are tested for each disease. Totally, the test data set contain 160 new samples.

\section{Experimental results}

Throughout this paper, we work on matrices of markers and samples from different types of leukemia that have been collected at Iran Blood Transfusion Organization (IBTO). The data set contains 400 samples taken from human leukemic bone marrow. It consists of 17 attributes, which determine different CD markers related to leukemia. Data from different experience stored in the form of matrices, rows referring to the CD markers (attributes) and columns show different samples of disease. All of the samples are of malignant class.

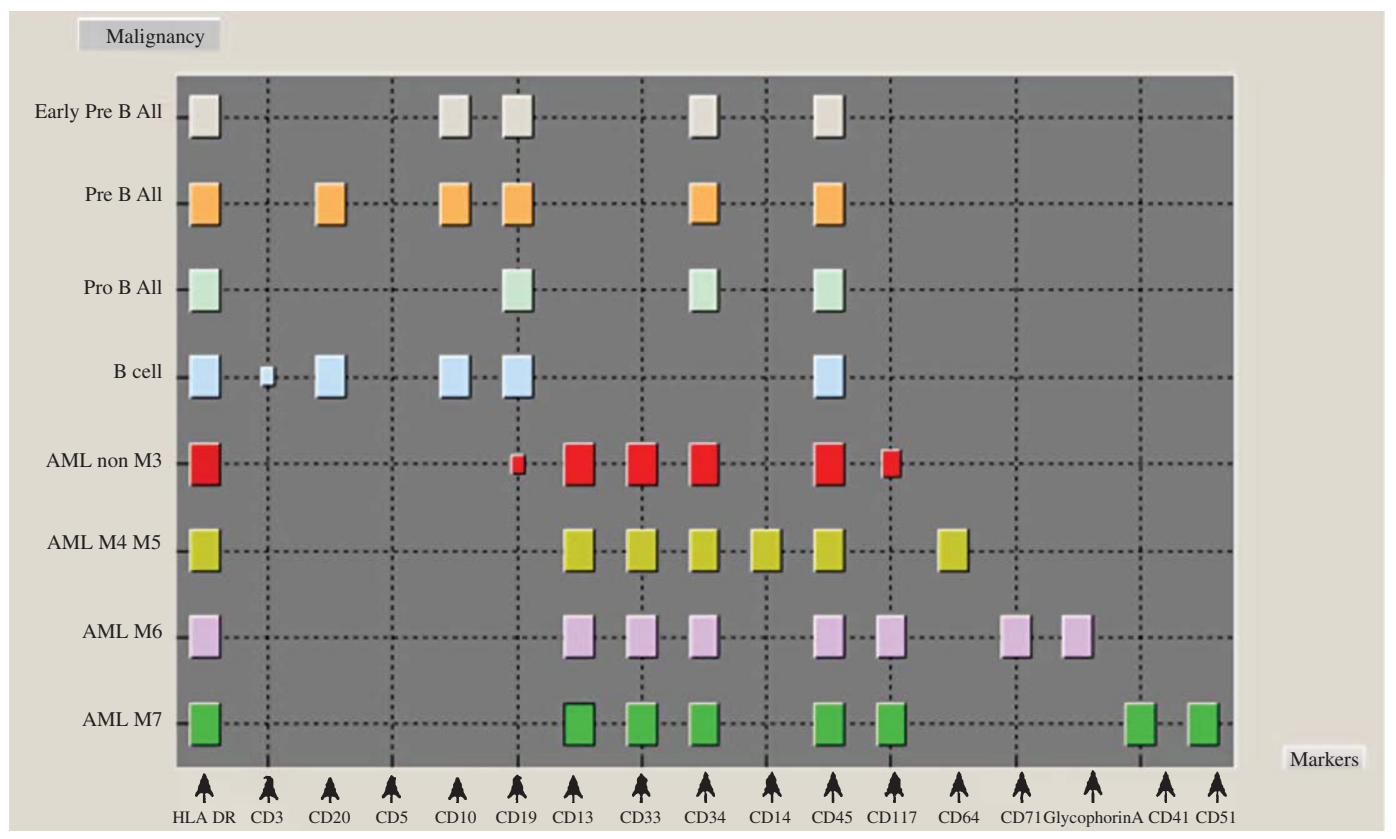

Fig. 5. Hinton Diagram showing weights. 
Table 2

Number of matching cases in proposed approach

\begin{tabular}{lcc}
\hline Diagnosis & $\begin{array}{c}\text { No. of Test } \\
\text { Samples }\end{array}$ & $\begin{array}{c}\text { No. of matching case } \\
\text { in classification }\end{array}$ \\
\hline Early Pre B All & 20 & $20(100 \%)$ \\
Pre B All & 20 & $15(75 \%)$ \\
Pro B All & 20 & $20(100 \%)$ \\
B Cell ALL & 20 & $20(100 \%)$ \\
Aml M4/M5 & 20 & $14(70 \%)$ \\
Aml M6 & 20 & $20(100 \%)$ \\
Aml M7 & 20 & $20(100 \%)$ \\
Aml Non M3 (M1M2) & 20 & $20(100 \%)$ \\
Total & 160 & $149(93.12 \%)$ \\
\hline
\end{tabular}

$60 \%$ of data have been used in Training phase while the remaining were used in testing phase. The Training and test data are separated from each other. As shown in Table 2, the average accuracy rate of the classification is $93.12 \%$. So, we can use the weights obtained from Shapley value for the best classification performance.

To assess the accuracy of the classification, it is useful to create a confusion matrix. In this matrix, the classification results are compared to additional truth information. Actually, a confusion matrix is a visualization tool which represents the nature of the classification errors, as well as their quantities. Table 3 is the confusion matrix that gives the number/proportion of test samples from one class classified in to another (or same) class.

The confusion matrix shows that the system can make the distinction between many types of malignancy pretty well. But, in 20 samples of the actual Pre B ALL, the system predicted that five were Early Pre B ALL, and of the $20 \mathrm{Aml} \mathrm{M} 4 / \mathrm{M} 5$, it predicted that 6 were Aml NonM3. It is observed from the matrix that the system has trouble in distinguishing between Pre B ALL, Early Pre B ALL and Aml M4/M5, Aml NonM3 because of their similarity in some markers. The distinction of AML non M3 from M4/M5 is difficult on morphology alone so $\mathrm{MPO}^{2}$ and $\mathrm{NSE}^{3}$ are needed for better classification. Also, in order to obtain more accurate classification, cytogenetic data are mandatory. The system can make the distinction between the other types of malignancy pretty well.

\footnotetext{
2 Myeloperoxidase (MPO).

${ }^{3}$ Non-specific esterase (NSE).
}

\subsection{Measures for performance evaluation}

To evaluate the efficiency of the proposed method, several measures such as classification accuracy, sensitivity and positive predictive value have been used. They are defined as follows;

Classification accuracy $(\%)=$

$$
\frac{T P+T N}{T P+F P+F N+T N}
$$

Sensitivity $(\%)=\frac{T P}{T P+F N} \times 100$

Positive predictive value $=\frac{T P}{T P+F P} \times 100$

Where TP is True Positive value, TN is True Negative value, FN is False Negative value, FP is False Positive value.

Sensitivity which is called as recall indicates the proposition of actual positives which are correctly identified. As it is shown, $93.12 \%$ of samples are correctly assigned to the right class. Positive predictive value which is called precision implies the capability of the proposed method in finding the correct disease.

The values of sensitivity, positive predictive value and classification accuracy for our system are shown in Table 4.

The F measure is the harmonic mean of the precision and recall. It is given as follow:

$$
F=\frac{2 \times \operatorname{Pr} \text { ecision } \times \operatorname{Re} \text { call }}{\operatorname{Pr} \text { ecision }+\operatorname{Re} \text { call }} \%
$$

We evaluate the overall performance of the search method in terms of the F measure. In this paper the F measure has been found $96.1 \%$.

\subsection{Comparison}

In order to compare our results, we attempt to implement the problem by one of the common classification algorithms. In this regards, decision tree (DT) is preferred. The DT classifier is one of the most popular machine learning techniques. In simple expression, DTs are classical tools helping to choose differentiation between several courses of action. They provide a highly effective structure within which you can lay out options and investigate the possible outcomes of 
Table 3

The Confusion Matrix

\begin{tabular}{|c|c|c|c|c|c|c|c|c|c|c|}
\hline & & \multicolumn{9}{|c|}{ Classification Result (predicted) } \\
\hline & & $\begin{array}{c}\text { Early Pre } \\
\text { B All }\end{array}$ & $\begin{array}{c}\text { Pre B } \\
\text { All }\end{array}$ & $\begin{array}{c}\text { Pro B } \\
\text { All }\end{array}$ & $\begin{array}{c}\text { B Cell } \\
\text { ALL }\end{array}$ & $\begin{array}{c}\text { Aml } \\
\text { M4 M5 }\end{array}$ & $\begin{array}{l}\text { Aml } \\
\text { M6 }\end{array}$ & $\begin{array}{l}\text { Aml } \\
\text { M7 }\end{array}$ & $\begin{array}{c}\text { Aml Non } \\
\text { M3 }\end{array}$ & Total \\
\hline & Early Pre B All & 20 & 0 & 0 & 0 & 0 & 0 & 0 & 0 & 20 \\
\hline & Pre B All & 5 & 15 & 0 & 0 & 0 & 0 & 0 & 0 & 20 \\
\hline & Pro B All & 0 & 0 & 20 & 0 & 0 & 0 & 0 & 0 & 20 \\
\hline Ground & B Cell ALL & 0 & 0 & 0 & 20 & 0 & 0 & 0 & 0 & 20 \\
\hline Truth & Aml M4 /M5 & 0 & 0 & 0 & 0 & 14 & 0 & 0 & 6 & 20 \\
\hline (Actual) & Aml M6 & 0 & 0 & 0 & 0 & 0 & 20 & 0 & 0 & 20 \\
\hline & Aml M7 & 0 & 0 & 0 & 0 & 0 & 0 & 20 & 0 & 20 \\
\hline & Aml Non M3 & 0 & 0 & 0 & 0 & 0 & 0 & 0 & 20 & 20 \\
\hline Total & & 25 & 15 & 20 & 20 & 14 & 20 & 20 & 26 & 240 \\
\hline
\end{tabular}

Table 4

Sensitivity, positive predictive value and classification accuracy for the proposed method

\begin{tabular}{ll}
\hline $\begin{array}{l}\text { Train-Test partition } \\
\text { Measures }\end{array}$ & 60-40\% Training/Test partition \\
\hline Sensitivity (\%) & 93.12 \\
Positive predictive value & 99.3 \\
Classification accuracy & 93.12 \\
\hline
\end{tabular}

choosing those options. A DT is made of decision and leaf nodes. Each decision node corresponds to a test X over a single attribute of the input data and has a number of branches, each of which handles an outcome of the test X. Each leaf node indicates as a class for a case. It shows the result of a decision for that case. [23]. Figure 6 shows a simple example of decision tree which displays the investigated leukemia in this paper. ${ }^{4}$

We employed Weka workbench to evaluate the results on Decision Tree. We have implemented one of the most common decision tree construction algorithms: C4.5 (called version J48). C4.5 algorithm is based on the ID3, with supplementary programming to address ID3 problems. ID3 is one the more famous Inductive Logic Programming methods, developed by Quinlan [18], an attribute based machine learning algorithm that creates a decision tree on a training set of data and an entropy measure to build the leaves of the tree [8].

C4.5 is an extension of the ID3 algorithm to address some issues (e.g., avoiding over fitting the data, decreased error pruning, handling continuous attributes and etc.) which are not encountered by ID3 [27].

\footnotetext{
${ }^{4}$ As previous mentioned, the AML M3 (APL) was not investigated because of few samples.
}

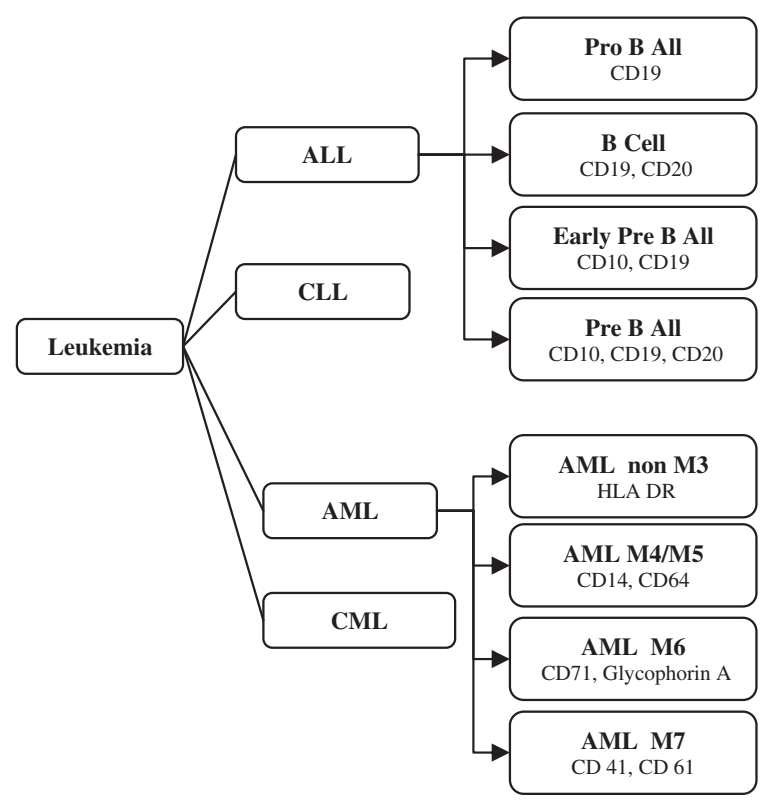

Fig. 6. Example of Decision Tree.

Table 5

Comparison the performance of presented system and Decision Tree

\begin{tabular}{ll}
\hline $\begin{array}{l}\text { Proposed System based on } \\
\text { cooperative game theory }\end{array}$ & 93.12 \\
Decision Tree (C4.5) & 90.16 \\
\hline
\end{tabular}

Again, $60 \%$ of the data set has been used in Training phase while the remaining were used in testing phase. The correct classification rate based on DT (C4.5) is depicted in Table 5 and compared with the result of proposed system based on cooperative game theory. 


\section{Concluding remarks}

Leukemia is a cancer which starts in hematopoietic elements primarily in the bone marrow, the soft tissue inside most bones. Over time, these cells can crowd out the normal blood cells, lymph nodes or other organs and lead to serious problems. Emphasis on diagnostic techniques and best treatments provide better prognosis and survival for patients. In this study, an A-MDSS for classifying leukemia based on cooperative game is presented. In our work, A-MDSS is used to prepare some useful suggestion of the type of leukemia to the clinical hematologist and could improve diagnostic accuracy which would result in more appropriate diagnosis and better survival. Actually, this system serves as basis platform on which to further iterations that could be meet a pathologist's diagnosis.

Throughout this paper, we worked on real data set from different types of leukemia that have been collected at Iran Blood Transfusion Organization (IBTO). The data set contains 400 samples taken from human leukemic bone marrow. The accuracy rate of classification based on cooperative game is $93.12 \%$. In the literature review, there were a little numbers of efforts for leukemia management. Each of those approaches used different types of data sets and diseases. To evaluate the performance of our proposed method based on cooperative game, we have applied DT (C4.5) technique. The accuracy rate of classification based on $\mathrm{C} 4.5$ is $90.16 \%$.

Additional performance measures such as Precision and Recall are also presented. The achieved rates for recall, precision and $\mathrm{F}$ measure are $93.12 \%, 99.3 \%$ 0.961, respectively. According to these results, it has been found that the proposed system gives very promising results in classifying leukemia. The approach is as a part of MDSS which could be very helpful to hematologists and physicians to make better decisions on their patients. According to this system the hematologist with her specialty can make better decision on her patient.

As the feature work, the development of this approach as a part of MDSS would be an interesting and challenging research perspective.

\section{Acknowledgments}

Special thanks to the Iran Blood Transfusion Organization (IBTO) for the information and data sets provided.

\section{References}

[1] M. Akay, Support vector machines combined with feature selection for breast cancer diagnosis, Expert Systems with Applications 36 (2009), 3240-3247.

[2] E. Alpaydin, Introduction to machine learning, The MIT Press 2004.

[3] L.A. Bacha, S.M. Bentzen, J. Alsnerb and F.B. Christiansena, An evolutionary-game model of tumour-cell interactions: Possible relevance to gene therapy, European Journal of Cancer 37 (2001), 2116-2120.

[4] N. Bellomo and M. Delitala, From the mathematical kinetic and stochastic game theory to modelling mutations, onset, progression and immune competition of cancer cells, Physics of Life Reviews 5 (2008), 183-206.

[5] E. Bollschweiler, S. Mönig, K. Hensler, E. Baldus, K. Maruyama and A. Hölscher, Artificial neural network for prediction of lymph node metastases in Gastric cancer: A phase II diagnostic study, Annals of Surgical Oncology 11 (2004), 506-511.

[6] A. Brandenburger, Cooperative Game Theory: Characteristic Functions, Allocations, Marginal Contribution, 2007.

[7] R. Brânzei, D. Dimitrov and S. Tijs, Models in Cooperative Game Theory, Springer 2008.

[8] V.P. Bresfelean, Analysis and Predictions on students' behavior using decision trees in Weka environment, 29th International Conference on Information Technology Interfaces, ITI 07 (2007), 51-56.

[9] Y.M. Chae, Q. Park, K.S. Park and M. Young, Development of medical decision support system for leukemia management, Expert system with applications 15 (1998), 309-315.

[10] C. Chang and C. Chen, Applying decision tree and neural network to increase quality of dermatologic diagnosis, Expert Systems with Applications 36 (2009), 4035-4041.

[11] S.M. Chou, T.S. Lee, Y.E. Shao and I.F. Chen, Mining the breast cancer pattern using artificial neural networks and multivariate adaptive regression splines, Expert Systems with Applications 27 (2004), 133-142.

[12] Fragnelli Vito and Moretti Stefano, A game theoretical approach to the classification problem in gene expression data analysis, Computers and Mathematics with Applications, 55 (2007), 950-959.

[13] J.M. Garcia-Gomez, C. Vidal, J. Vicente, L. Marti-Bonmati and M. Robles, Medical decision support system for diagnosis of soft tissue tumors based on distributed architecture, 26th Annual International Conference of the IEEE EMBS (2004).

[14] M.J. Horner, Ries Lag, M. Krapcho, N. Neyman, R. Aminou, N. Howlader, S.F. Altekruse, E.J. Feuer, L. Huang, A. Mariotto, B.A. Miller, D.R. Lewis, M.P. Eisner, D.G. Stinchcomb, B.K. Edwards (Eds), Seer Cancer Statistics Review1975-2006, National Cancer Institute, Bethesda MD. http://seer.cancer. gov/csr/1975_2006/ based on November 2008 SEER data submission posted to the SEER web site 2009.

[15] M. Karabatak and M. Cevdet, An expert system for detection of breast cancer based on association rules and neural network, Expert Systems with Applications 36 (2009), 3465-3469.

[16] Y. Mansury, M. Diggory and T. Deisboeck, Evolutionary game theory in an agent-based brain tumor model: Exploring the 'Genotype-Phenotype'link, Journal of Theoretical Biology 238 (2006), 146-156. 
[17] D. Michie, D.J. Spiegelhalter and C.C. Tayor, Machine learning, neural and statistical classification, Ellis Horwood, London, 1994.

[18] J. Quinlan, Induction of decision trees, Machine Learning, Morgan Kaufmann 1986, 81-106.

[19] S. Sahan, K. Polat, H. Kodaz and S. Günes, A new hybrid method based on fuzzy-artificial immune system and k-nn algorithm for breast cancer diagnosis, Computers in Biology and Medicine 37 (2007), 415-423.

[20] I. Sefion, M. Gailhardou and A. Ennaji, A Medical Decision Support System for Asthmatic Patient Health Care, Artificial Intelligence and Applications (AIA) (2002), 362-344.

[21] M. Sewak, P. Vaida, C. Chan and Z. Duan, SVM Approach to Breast cancer classification, Second International Multisymposium on Computer and Computational Sciences (2007).

[22] L. Shapley, A value for n-person games, in: H.W. Kuhn, A.W. Tucker (eds.), Contributions to the theory of games II, Annals of Mathematics Studies 28 (1953), 307-317.
[23] S. Sheen and R. Rajesh, Network Intrusion Detection using Feature Selection and Decision tree classifier, IEEE Region 10 Conference, TENCON 08 (2008), 1-4.

[24] T. Shusaku, Web Based Medical Decision Support System for Neurological diseases, IEEE/WIC International Conference on Web Intelligence 629 (2003).

[25] M. Skapura David, Building neural networks, Addison Wesley 1996.

[26] A. Torkaman, N. Moghaddam Charkari, M. Aghaeipour and E. Hajati, A recommender system for detection of leukemia based on cooperative game, 17th Mediterranean Conference on Control and Automation (MED' 09) (2009). Thessaloniki Greece.

[27] P.H. Winston, Artificial Intelligence, Addison Wesley 1992.

[28] B. Wood, M. Borowitz, N. Abraham, H. Massey, M. Bluth, J. Miller, G. Threatte, R. Hutchison, E. Unger and M. Lifshitz, Henry's Clinical Diagnosis And Management by Laboratory Methods (2007). 


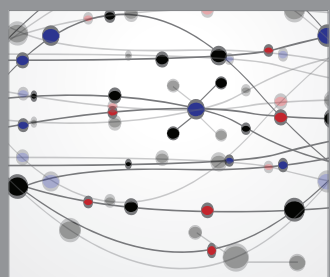

The Scientific World Journal
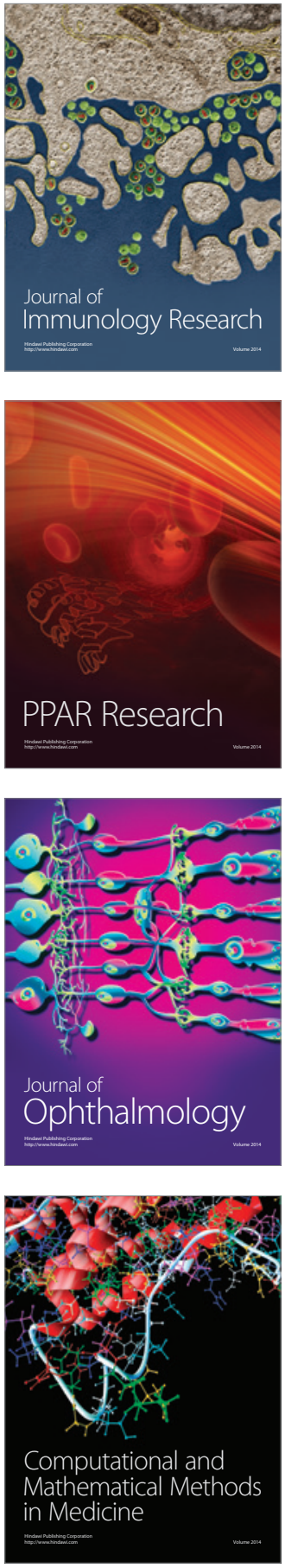

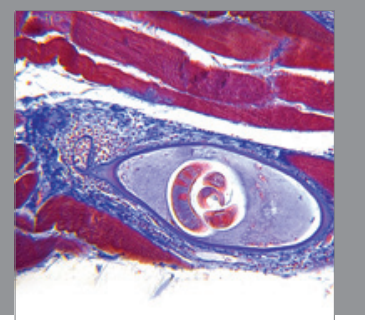

Gastroenterology

Research and Practice
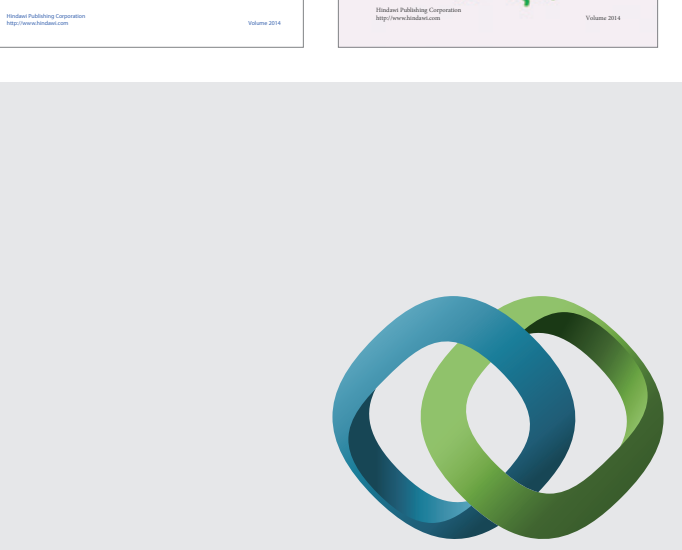

\section{Hindawi}

Submit your manuscripts at

http://www.hindawi.com
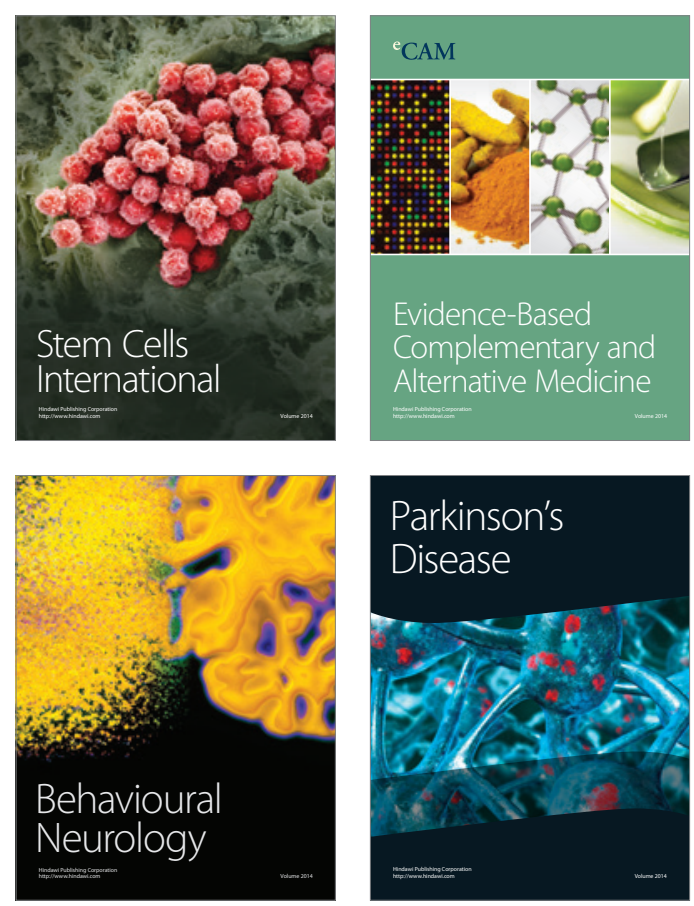

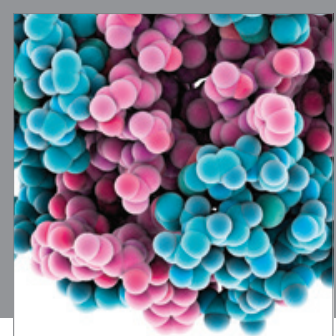

Journal of
Diabetes Research

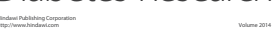

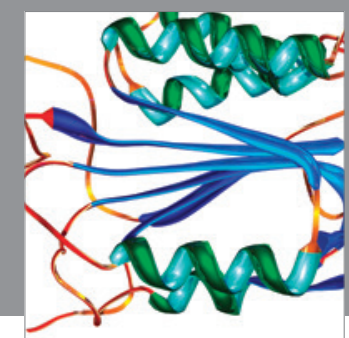

Disease Markers
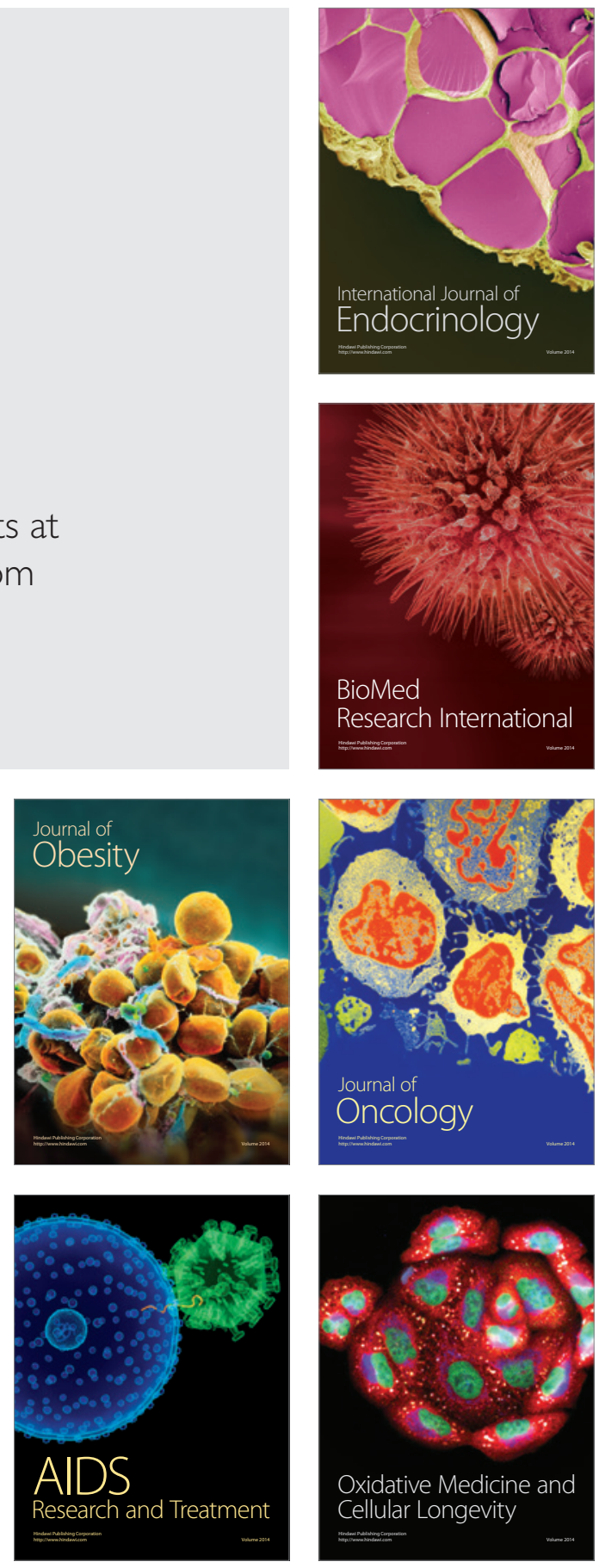